Original Article

\title{
Violent behavior patterns: possible homeopathic remedies
}

\author{
Célia Regina Barollo (MD)1,2, Fernando Antônio Cardoso Bignardi (MD) ${ }^{1,2}$,

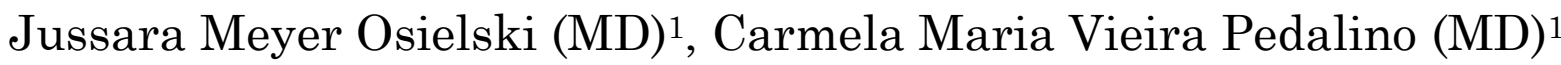

\author{
${ }^{1}$ NEPHSP - Núcleo de Estudos e Pesquisas em Homeopatia, São Paulo, Brazil \\ ${ }^{2}$ Centro de Estudos do Envelhecimento/UNIFESP, São Paulo, Brazil
}

\begin{abstract}
The authors discuss violence as a current epidemics and violent behavior in children and adolescents. They present a repertory study including the characteristic and peculiar symptoms of 12 patients, with clinical diagnoses of Attention Deficit Hyperactivity Disorder (ADHD), Conduct Disorder and Oppositional Defiant Disorder, and the symptoms corresponding to the diagnostic criteria in the DSM-IV transcribed in repertory language. The result is a materia medica for patterns of violent behavior.
\end{abstract}

Keywords: violent behavior, homeopathy, children and adolescents, ADHD, Conduct Disorder, Oppositional Defiant Disorder.

\section{Introduction}

Violence is a pattern of human behavior characterized by anger, frequently associated to actual or potential physical aggression in order to obtain something. "Violence results from the action or an irresistible power in order to achieve an objective that would not have been achieved without it"[1]. Masi Elizalde [2] utilizes the terminology egolysis and alterlysis to name miasmatic dynamics stages related to auto (against oneself), hetero (against others) and eco violence (against environment) [3].

Following a worldwide tendency, violence is also increasing in Brazil. Although it is often attributed to violent male outlaw teenagers [4-7], in fact a relatively small number of youths commit a large part of crimes. Violent behavior is not exclusive of socially marginalized individuals and is not always related to socioeconomic status [8, 9]. Children and teenagers, in whom the mind and brain formative process is not yet finished, have difficulty to consider the limits between the real and virtual worlds, thus, beyond environmental socioeconomic conditions, violence presented by the media can act like a trigger in susceptible individuals to promote violent behavior [10-12]. This fact is also observed in clinical homeopathic practice, when pathological stimuli and susceptibility are synergic.

Children and adolescents in violent settings may exhibit patterns of violent behavior frequently associated to Attention Deficit Disorder (ADD), with or without Hyperactivity / Impulsiveness (ADHDI) $[13,14]$. ADD, a neuropsychological disturbance that alters the ability to concentrate in tasks and to control impulses and motor activity has become the most frequent health problems in children and one of the main causes of learning disturbance and school failure. It affects around $20 \%$ of children in school age and attacks three times more males than females. It usually begins before the age of 7 , might continue throughout adolescence to reach adulthood. On the other hand, learning disturbance may be a risk factor for delinquent behavior [15-17].

In the DSM-IV section devoted to Conduct Disorder (CD, CID-10 312,8) and Oppositional Defiant Disorder (ODD, CID-10 313.81) [18], there are listed and classified the diagnostic criteria of behavioral 
disturbs related to children and adolescents unlawful acts [19]. They can be compared to the symptoms contained in the homeopathic repertory [20] and related to the symptoms of egolysis and alterlysis.

The standard treatment of these conditions uses methylphenidate $\left(\operatorname{Ritalin}^{\circledR}\right)$ or amphetamine, which may cause the same problems they are able to treat: lack of attention, hyperactivity and impulsiveness. Moreover, hyperactive children taking methylphenidate are at a higher risk of developing an addiction. It may cause the same devastating effects as prolonged use of cocaine [21]. Thus, the search for alternative therapeutic options is justified; homeopathy may be one of them.

\section{An epidemics of violence?}

Currently, an epidemics is defined as the incidence in a short period of time of a large number of cases of a disease. But, in a more comprehensive concept, it can be defined as an abnormal situation in a community, a potential severe event triggering social pressures that must be answered by the sanitary authorities with the highest urgency.

Epidemiology means the "study of the epidemics" and is defined as the study of the distribution and determinants of the states or events related to health in a specific population. The application of these studies is used to control health problems (Center for Disease Center/Atlanta-USA). The epidemiology of infectious diseases is still of highest importance, but together with the perception that several factors contribute to the rise of any one disease, epidemiologists are also approaching chronic illnesses. As an area in constant transformation [22] it is expanding into other fields of knowledge like sociology and law. According to Barata [23], the understanding of the process of health-illness has improved with the emergence of social epidemiology, which investigates the social determinants of disease. Thus, some authors consider violence as a modern epidemics, threatening populations not only physically, but in its basic institutions like the family and the work and school environments [24-33].

Although violent behavior is associated to many causes, including biological, metabolic, economic, cultural and social conditions, family dynamics, spiritual conditions, experienced trauma, etc., in many cases the contact ("contagion") with or by a violent environment can awake in the susceptible this kind of behavior, which would not occur in its absence, exactly as in the case of infectious biological agents.

It may sound odd to speak of an "epidemics of violence", due to the current concept of epidemics, usually associated to the occurrence of an acute infectious disease involving "contagion/infection". However, it is only a part of children and adolescents living in violent environments who become "infected" by violence - precisely those children susceptible to this "contagion". From a classic homeopathy point of view, it could be spoken as an "energy contagion", as Kent stated [34], or a change in the miasmatic dynamics [2].

The aim of this study is to define homeopathic remedies useful in violent situations affecting children and adolescents.

\section{Materials and Methods}

From the records of 12 children and adolescents homeopathically treated for CD, ODD, ADD and ADHDI $[3,14,35,36]$ the peculiar and most characteristic symptoms were extracted. The list was completed with the diagnostic criteria for these same disorders as described in DSM-IV transcribed into the homeopathic repertory language.

All these symptoms were analyzed together [20,37], as if they belonged to one only patient in order to characterize the symptomatic image of the epidemics. The resulting remedies were classified in decreasing value of coverage, in order to compose a list of the remedies that when tested on healthy human beings showed a potential to elicit violent acts, so to be applied by similarity to actual patients; 2) to facilitate the differential diagnosis among them in real-time practice and guide the search in the homeopathic materia medica [38-45].

\section{Results and discussion}

The repertory analysis of the homeopathic symptoms corresponding to ADHDI, CD and ODD is presented in Table 1 . The 25 remedies with the highest potential to elicit the characteristic symptoms of these disturbs on healthy individuals are: Aconitum napellus, Agaricus, Anacardium orientale, Arsenicum album, Belladona, Bufo rana, Calcarea carbonica, Causticum, Chamomilla, Hepar sulphur, Hyosciamus niger, Lachesis muta, Lycopodium clavatum, Mercurius, Nitric acidum, Nux vomica, Opium, Phosphorus, Plumbum, Staphisagria, Stramonium, Sulphur, Tarentula, Veratrum album e Zincum.

Although some authors [46-49] have described other remedies as violent, they were not included in our list as they did not present a significant part of the selected symptoms: Absinthum, Alcoholum, Aurum metallicum, Luesinum, Platina and Sepia succus.

The compared Materia Medica of violent behavior patterns is presented in Table 2 . 


\section{References}

[1] Silva DP. Vocabulário jurídico. 11 $11^{\text {st }}$ ed. Rio de Janeiro: Forense; 1989.

[2] Masi Elizalde, A. Actas del Instituto de Altos Estudios Homoeopáticos "James Tyler Kent", Buenos Aires; Club de Lectores: 1988.

[3] Barollo CR, et al. Efeito da homeopatia no tratamento de crianças e adolescentes em situação de violência. Cultura Homeopática. 2007; 21: 5-10.

[4] Secretaria da Segurança Pública. SSP-SP Levantamento completo sobre os principais tipos de crimes cometidos exclusivamente por crianças e adolescentes no Estado de São Paulo. São Paulo: SSP; 2001.

[5] SEDH; Levantamento da Subsecretaria de Promoção dos Direitos da Criança e do Adolescente/ SEDH, baseado em informações fornecidas pelos estados, Comparação entre 2004 e 2006. São Paulo: SEHD; 2007.

[6] Toledo GW. A delinqüência juvenil no Estado de São Paulo: características, evolução e tendências observadas entre os anos de 1950, 1960, 1979, 1985, 1995, 2000, 2001 e 2002 [Dissertation (Master)]. Ribeirão Preto: Faculdade de Filosofia, Ciências e Letras de Ribeirão Preto (FFCLRP); 2006.

[7] Adorno S, Bordini EBT; Lima RS. O adolescente e as mudanças na criminalidade urbana. São Paulo perspect. 1999 ; 13(4): 62-74

[8] Mcfadyen-Ketchum S, Bates JE, Dodge KAY, Pettit, GS. Patterns of change in early childhood aggressive-disruptive behavior: gender differences in predictions from early coercive and affectionate mother-child interactions. Child Dev. 1996; 67: 2417-2433.

[9] Gallo AE, Williams LCA. Adolescentes em conflito com a lei: uma revisão dos fatores de risco para a conduta infracional. Psicol Teor Prat. 2005; 7(1): 81-95.

[10] Caspi A, et al. Role of genotype in the cycle of violence in maltreated children. Science. 2002; 297: 851-854.

[11] Miczek KA; Almeida RMM. Neurobiology of escalated aggression and violence. J Neurosci. 2007; 27: 11803-11806.

[12] Nelson RJ; Trainor BC. Neural mechanisms of aggression. Nature Reviews. Neuroscience. 2007; 8: 536-546.

[13] Biederman J, Munir KY, Knee D. Conduct and oppositional disorder in clinically referred children with attention deficit disorder: a controlled family study. J American Academy of Child and Adolescent Psyc. 1987; 26: 724-727.

[14] Barollo CR, Alves D, Bignardi FAC, Décourt I, Freitas Jr V, Hubner V, et al. Efeito do tratamento homeopático sobre o comportamento do adoecer crônico, em uma comunidade carente, alvo de programa social, na periferia da cidade de São Paulo, no período de ago/2004 a mar/2006. Proceedings of the XXVIII Congresso Brasileiro de Homeopatia; 2006 Sep 03-07; Florianópolis, Brasil. Florianópolis: Associação Médica Homeopática de Santa Catarina; 2006. 86-97.

[15] Dias MDF. Fatores de risco para o comportamento dos infratores [Dissertation (Master)]. São Paulo: Universidade Federal de São Paulo, UNIFESP; 2002.

[16] Doreleijers TAH, Prins-Aardema C. Distúrbios de aprendizagem em delinqüentes juvenis: investigação científica, projectos de tratamento e de prevenção na Holanda. Rev Port Pedag. 2000; 34(2): 303-321.

[17] Oliveira, CA. A fuga da escola como preditor do fenômeno delinqüencial / The escape from school as a cause of the delinquency phenomenon. Aletheia. 2002; 15: 27-36.

[18] Jorge, Miguel R; DSM IV - Manual Diagnóstico e Estatístico de Transtornos Mentais. $4^{\text {th }}$ ed. Porto Alegre: Artmed; 2000.

[19] Cury M, Silva AFA, Mendez EG. Estatuto da Criança e do Adolescente comentado. $5^{\text {th }}$ ed. São Paulo: Malheiros; 2002.

[20] Ribeiro Filho A. Repertório de homeopatia. São Paulo: Organon; 2005.

[21] Volkow ND. Therapeutic doses of oral methylphenidate significantly increase extracellular dopamine in the human brain. J Neurosci. 2001; 21: RC121.

[22] Mattar A, Ivankovich DT, da Cunha FM, Abdal LHS. Internet e Epidem. [cited 2008 Jan 28]. Available from:

http://www.virtual.epm.br/material/tis/currmed/temas/med5/med5t41999/dado/dadomepid.htm.

[23] Barata RB. Epidemiologia social. Rev Bras Epidemiol. 2005; 8(1): 7-17

[24] Alves Sobrinho EJM, Inojosa RM. Gestão social nos municípios: a violência e a cultura de paz. Rev Adm Pública. 2005; 39(2): 279-295.

[25] Carvalheiro JR. Editorial: Violent deaths: the epidemic of the third millenium? Rev Bras Epidemiol. 1999; 2(3): 99-101. 
[26] Chaui M. Cultura política e política cultural. Estud Av. 1995; 9(23):71-84.

[27] Minayo MCS. Health affairs: The quarterly journal of the health sphere. Cad Saúde Pública. 1994; 10(3): 401-403.

[28] Minayo MCS, Souza ER. Violência e saúde como um campo interdisciplinar e de ação coletiva. História. Ciências, Saúde - Manguinhos. 1997-1998; 4(3): 513-531.

[29] Minayo MCS, Souza ER. Is it possible to prevent violence? Reflections in public health area. Ciênc Saúde Coletiva. 1999; 4(1): 7-23.

[30] Silva SF. Crescimento da violência urbana: as grandes cidades estão diante de uma epidemia social? Divulg Saúde Debate. 2004; (30): 10-14.

[31] Steiner JE. Conhecimento: gargalos para um Brasil no futuro. Estud Av. 2006; 20(56): 75-90.

[32] Szwarcwald CL, Castilho EA. Mortalidade por armas de fogo no estado do Rio de Janeiro, Brasil: uma análise espacial. Rev Panam Salud Publica. 1998; 4(3): 161-170.

[33] Taborda JGV. Temas em psiquiatria forense e psicologia jurídica. Rev Bras Psiquiatr. 2003; 25(3): 194-194.

[34] Kent JT. Lições de filosofia homeopática. $2^{\text {nd }}$ ed. São Paulo: Organon; 2002.

[35] Barollo CR. O efeito da homeopatia na dinâmica miasmática de crianças em situação de violência: casos clínicos. Proceedings of the II Encontro Internacional em Latinoamerica Alfonso Masi Elizalde; 2007 Sep 14-16; Buenos Aires, Argentina. Buenos Aires: Inistitulo de Altos Estudios Homeopáticos James Tyler Kent; 2007 54-63.

[36] Barollo CR. Clinical cases: childhood enuresis and serious syndrome from social disagreement. Proceedings of the I Simpósio Internazionale FIAMO Brasil-Itália; 2007 Nov 16-18; Rome, Italy. São Paulo: Associação Paulista de Homeopatia; 2007. 45-67.
[37] Schroyens F. Synthesis/ RADAR homeopathic software version. 7.3. United Kingdom: Philip Edmonds DSH RSHom; 2006.

[38] Boericke W. Homœopathic materia medica [book online]. New Delhi: B Jain. [cited 2008 Feb 02]. Available from: http://homeoint.org/books/boericmm/index.htm.

[39] Deltombe M, Jaegerschmid G. Matéria médica homeopática em pediatria. São Paulo: Andrei; 1996.

[40] Kent JT. Lectures on Homœopathic materia medica. New Delhi: B Jain. [cited 2008 Feb 02]. Available

http://homeoint.org/books3/kentmm/index.htm.

[41] Lamothe J. Homeopatia pediátrica. São Paulo: Andrei; 1999.

[42] Lippe A. Textbook of materia medica. Kent: WB Saunders Company. [cited 2008 Feb 02]. Available from:

http://homeoint.org/books1/lippemm/index.htm.

[43] Masi Elizalde. A. Jornada Paulista de Homeopatia. São Paulo: Associação Paulista de Homeopatia; 1999 Nov.

[44] Masi Elizalde A. Jornadas de Matéria Médica. Rio de Janeiro: Escola Kentiana do Rio de Janeiro; 2000.

[45] Vijnovsky B. Tratado de matéria médica homeopática. São Paulo: Organon; 2005.

[46] Papaphilipppou G. Violent remedies. Homoepathic Links. 1991; 3: 17-19.

[47] Picovsky E. La violência en la matéria médica. Proceedings of the Jornada de Medicina Homeopática del Uruguai; 1993 Dec; Montevideo, Uruguay. 1993. 19-23.

[48] Casale JA. Rubros de la violencia extrema. Homeopatia. 1994; 59: 221-222.

[49] Barrault M. Violence et sociopathie de l'adolescent. Cah Biothérapie. 1999; 157: 38-40.

\section{(c) BY-NC-ND Licensed to GIRI}

Support: author declares that this study received no funding

Conflict of interest: authors declare there is no conflict of interest

Received: 15 October 2007; Revised: 10 February 2008; Published: 31 March 2008

Erratum: 30 Dec 2008. (http://www.feg.unesp.br/ ojs/zacha_ijhdr/erratum/?v=7\&i=22\&pi=7)

Correspondence author: Célia R. Barollo, crbarollo@ajato.com.br; http://www.nephsp.org

How to cite this article: Barollo CR, Bignardi FAC, Osielski JM, Pedalino CMV.Violent behavior patterns: possible homeopathic remedies. Int J High Dilution Res [online]. 2008 [cited YYYY Mmm DD]; 7(22):7-21. Available from: http://www.feg.unesp.br/ ojs/index.php/ijhdr/article/view/257/341. 
Table 1. Repertory analysis of ADHDI, CD and ODD homeopathic symptoms

\begin{tabular}{|c|c|c|c|c|c|c|c|c|c|c|c|c|c|c|c|c|c|c|c|c|c|c|c|c|c|c|}
\hline $\begin{array}{l}\text { Remedies/ } \\
\text { Symptoms }\end{array}$ & $\frac{\tilde{\pi}}{\tilde{\pi}}$ & 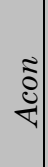 & \begin{tabular}{l}
$\bar{z}$ \\
$\vdots$ \\
\hdashline
\end{tabular} & 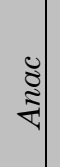 & $\stackrel{\infty}{\overline{4}}$ & $\underset{\widetilde{\Xi}}{\approx}$ & ำ & ت̃ & ర్య & 离 & $\stackrel{ఖ}{\beth}$ & : & 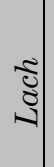 & ลี่ & : & 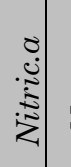 & 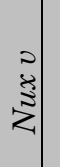 & 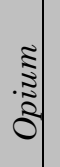 & 定 & 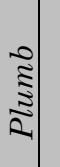 & 离 & $\begin{array}{c}5 \\
\vdots \\
\overline{5} \\
\omega\end{array}$ & $\frac{5}{5}$ & 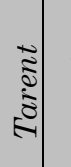 & $\begin{array}{l}\tilde{0} \\
\tilde{s} \\
\vdots\end{array}$ & 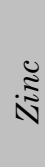 \\
\hline \multicolumn{27}{|c|}{ READING DISORDERS (IDC - 315.0) } \\
\hline $\begin{array}{l}\text { Concentration } \\
\text { difficult }\end{array}$ & & - & - & - & - & - & - & - & - & - & - & $\mathbf{x}$ & - & - & - & - & - & - & $\mathbf{x}$ & - & - & - & - & - & $\mathbf{x}$ & - \\
\hline $\begin{array}{l}\text { Concentration } \\
\text { difficult, studying }\end{array}$ & & $\mathbf{x}$ & $\mathbf{x}$ & - & - & $\mathbf{x}$ & - & - & $\mathbf{x}$ & $\mathbf{x}$ & - & - & $\mathbf{x}$ & $\mathbf{x}$ & $\mathbf{x}$ & - & $\mathbf{x}$ & - & - & - & $\mathbf{x}$ & - & $\mathbf{x}$ & - & - & - \\
\hline $\begin{array}{l}\text { Unable to read }+ \\
\text { mistakes reading+ } \\
\text { confusion of mind, } \\
\text { reading }\end{array}$ & & $\mathbf{x}$ & $\mathbf{x}$ & - & - & $\mathbf{x}$ & - & $\mathbf{x}$ & $\mathbf{x}$ & $\mathbf{x}$ & - & $\mathbf{x}$ & $\mathbf{x}$ & $\mathbf{x}$ & $\mathbf{x}$ & - & $\mathbf{x}$ & - & - & $\mathbf{x}$ & $\mathbf{x}$ & - & $\mathbf{x}$ & - & - & - \\
\hline \multicolumn{27}{|c|}{ MATHEMATICS DISORDERS (IDC - 315.1) } \\
\hline $\begin{array}{l}\text { Concentration } \\
\text { difficult calculating }\end{array}$ & & - & - & - & - & - & - & - & - & - & - & - & - & $\mathbf{x}$ & $\mathbf{x}$ & - & $\mathbf{x}$ & - & - & - & - & - & - & - & - & - \\
\hline $\begin{array}{l}\text { Inability for } \\
\text { mathematics }+ \\
\text { calculating } \\
+ \text { algebra }+ \\
\text { calculating mistakes }\end{array}$ & & - & - & - & - & $\mathbf{x}$ & - & $\mathbf{x}$ & $\mathbf{x}$ & - & - & - & $\mathbf{x}$ & $\mathbf{x}$ & $\mathbf{x}$ & - & $\mathbf{x}$ & - & - & - & $\mathbf{x}$ & - & - & - & - & - \\
\hline \multicolumn{27}{|c|}{ WRITING EXPRESSION DISORDER (IDC - 315.2) } \\
\hline $\begin{array}{l}\text { Difficult concentration } \\
\text { while writing }\end{array}$ & & $\mathbf{x}$ & - & - & - & - & - & - & - & - & - & - & - & - & $\mathbf{x}$ & - & - & - & - & - & - & - & - & - & - & - \\
\hline $\begin{array}{l}\text { Inability for writing }+ \\
\text { mistakes writing }+ \\
\text { confusion while } \\
\text { writing }\end{array}$ & & - & $\mathbf{x}$ & - & $\mathbf{x}$ & - & - & - & $\mathbf{x}$ & $\mathbf{x}$ & $\mathbf{x}$ & - & $\mathbf{x}$ & $\mathbf{x}$ & $\mathbf{x}$ & - & $\mathbf{x}$ & - & $\mathbf{x}$ & - & $\mathbf{x}$ & $\mathbf{x}$ & $\mathbf{x}$ & - & - & $\mathbf{x}$ \\
\hline \multicolumn{27}{|c|}{ SPEECH EXPRESSION DISORDER (IDC - 314.31) } \\
\hline Loquacity & & $\mathbf{x}$ & $\mathbf{x}$ & $\mathbf{x}$ & $\mathbf{x}$ & $\mathbf{x}$ & $\mathbf{x}$ & $\mathbf{x}$ & $\mathbf{x}$ & - & $\mathbf{x}$ & $\mathbf{x}$ & $\mathbf{x}$ & $\mathbf{x}$ & - & - & $\mathbf{x}$ & $\mathbf{x}$ & $\mathbf{x} \mid \mathbf{x}$ & $\mathbf{x}$ & $\mathbf{x}$ & $\mathbf{x}$ & $\mathbf{x}$ & $\mathbf{x} \mid \mathbf{x}$ & $\mathbf{x}$ & $\mathbf{x}$ \\
\hline Mistakes speaking & & - & $\mathbf{x}$ & - & - & $\mathbf{x}$ & $\mathbf{x}$ & $\mathbf{x}$ & $\mathbf{x}$ & $\mathbf{x}$ & $\mathbf{x}$ & $\mathbf{x}$ & $\mathbf{x}$ & $\mathbf{x}$ & $\mathbf{x}$ & - & $\mathbf{x}$ & $\mathbf{x}$ & - & - & $\mathbf{x}$ & $\mathbf{x}$ & $\mathbf{x}$ & - & - & $\mathbf{x}$ \\
\hline $\begin{array}{l}\text { Stammering (IDC - } \\
307.0)\end{array}$ & & $\mathbf{x}$ & $\mathbf{x}$ & - & $\mathbf{x}$ & $\mathbf{x}$ & $\mathbf{x}$ & & $\mathbf{x}$ & $\mathbf{x}$ & - & $\mathbf{x}$ & $\mathbf{x}$ & & $\mathbf{x}$ & - & $\mathbf{x}$ & $\mathbf{x}$ & $\mathbf{x}$ & $\mathbf{x}$ & - & $\mathbf{x}$ & $\mathbf{x}$ & - & $\mathbf{x}$ & - \\
\hline Speech confused & & - & $\mathbf{x}$ & - & - & $\mathbf{x}$ & $\mathbf{x}$ & $\mathbf{x}$ & $\mathbf{x}$ & $\mathbf{x}$ & $\mathbf{x}$ & $\mathbf{x}$ & $\mathbf{x}$ & $\mathbf{x}$ & $\mathbf{x}$ & - & $\mathbf{x}$ & $\mathbf{x}$ & - & - & $\mathbf{x}$ & $\mathbf{x}$ & $\mathbf{x}$ & - & - & $\mathbf{x}$ \\
\hline
\end{tabular}




\begin{tabular}{|c|c|c|c|c|c|c|c|c|c|c|c|c|c|c|c|c|c|c|c|c|c|c|c|c|c|}
\hline Absentminded & $\mathbf{x}$ & $\mathbf{x}$ & $\mathbf{x}$ & $\mathbf{x}$ & $\mathbf{x}$ & $\mathbf{x}$ & $\mathbf{x}$ & $\mathbf{x}$ & $\mathbf{x}$ & $\mathbf{x}$ & $\mathbf{x}$ & $\mathbf{x}$ & $\mathbf{x}$ & $\mathbf{x}$ & $\mathbf{x}$ & $\mathbf{x}$ & $\mathbf{x}$ & $\mathbf{x}$ & $\mathbf{x}$ & $\mathbf{x}$ & $\mathbf{x}$ & $\mathbf{x}$ & $\mathbf{x}$ & $\mathbf{x}$ & $\mathbf{x}$ \\
\hline Awkward & - & $\mathbf{x}$ & $\mathbf{x}$ & - & - & $\mathbf{x}$ & $\mathbf{x}$ & $\mathbf{x}$ & - & $\mathbf{x}$ & $\mathbf{x}$ & $\mathbf{x}$ & $\mathbf{x}$ & - & - & $\mathbf{x}$ & $\mathbf{x}$ & $\mathbf{x}$ & $\mathbf{x}$ & $\mathbf{x}$ & $\mathbf{x}$ & $\mathbf{x}$ & $\mathbf{x}$ & $\mathbf{x}$ & - \\
\hline $\begin{array}{l}\text { Difficult concentration } \\
\text { while studying }\end{array}$ & $\mathbf{x}$ & $\mathbf{x}$ & $\mathbf{x}$ & $\mathbf{x}$ & $\mathbf{x}$ & - & - & $\mathbf{x}$ & $\mathbf{x}$ & - & - & $\mathbf{x}$ & $\mathbf{x}$ & $\mathbf{x}$ & - & $\mathbf{x}$ & - & - & - & $\mathbf{x}$ & - & $\mathbf{x}$ & - & - & - \\
\hline Forgetful & $\mathbf{x}$ & $\mathbf{x}$ & $\mathbf{x}$ & $\mathbf{x}$ & $\mathbf{x}$ & - & $\mathbf{x}$ & $\mathbf{x}$ & $\mathbf{x}$ & $\mathbf{x}$ & $\mathbf{x}$ & $\mathbf{x}$ & $\mathbf{x}$ & $\mathbf{x}$ & $\mathbf{x}$ & $\mathbf{x}$ & $\mathbf{x}$ & $\mathbf{x}$ & $\mathbf{x}$ & $\mathbf{x}$ & $\mathbf{x}$ & $\mathbf{x}$ & - & $\mathbf{x}$ & $\mathbf{x}$ \\
\hline Muddled & $\mathbf{x}$ & $\mathbf{x}$ & $\mathbf{x}$ & $\mathbf{x}$ & $\mathbf{x}$ & - & - & - & - & - & - & $\mathbf{x}$ & - & $\mathbf{x}$ & - & $\mathbf{x}$ & - & $\mathbf{x}$ & - & $\mathbf{x}$ & - & - & - & - & $\mathbf{x}$ \\
\hline \multicolumn{26}{|c|}{ WITH HIPERATIVITY } \\
\hline Climb, desire to & - & - & - & - & $\mathbf{x}$ & - & - & - & - & - & $\mathbf{x}$ & - & - & - & - & - & - & - & - & - & $\mathbf{x}$ & - & - & - & - \\
\hline Dancing & $\mathbf{x}$ & $\mathbf{x}$ & - & - & $\mathbf{x}$ & - & - & $\mathbf{x}$ & - & - & $\mathbf{x}$ & $\mathbf{x}$ & & $\mathbf{x}$ & - & - & - & - & - & - & $\mathbf{x}$ & - & $\mathbf{x}$ & - & - \\
\hline $\begin{array}{l}\text { Restlessness in } \\
\text { children }\end{array}$ & $\mathbf{x}$ & $\mathbf{x}$ & $\mathbf{x}$ & $\mathbf{x}$ & $\mathbf{x}$ & $\mathbf{x}$ & - & $\mathbf{x}$ & $\mathbf{x}$ & $\mathbf{x}$ & $\mathbf{x}$ & $\mathbf{x}$ & & $\mathbf{x}$ & $\mathbf{x}$ & $\mathbf{x}$ & - & - & $\mathbf{x}$ & $\mathbf{x}$ & $\mathbf{x}$ & $\mathbf{x}$ & $\mathbf{x}$ & $\mathbf{x}$ & $\mathbf{x}$ \\
\hline $\begin{array}{l}\text { Restlessness - arms } \\
\text { and hands }\end{array}$ & - & - & - & $\mathbf{x}$ & $\mathbf{x}$ & & $\mathbf{x}$ & - & - & - & $\mathbf{x}$ & - & $\mathbf{x}$ & - & - & - & - & $\mathbf{x}$ & $\mathbf{x}$ & - & $\mathbf{x}$ & - & $\mathbf{x}$ & - & - \\
\hline $\begin{array}{l}\text { Restlessness - legs } \\
\text { and feet }\end{array}$ & - & $\mathbf{x}$ & - & $\mathbf{x}$ & - & $\mathbf{x}$ & - & $\mathbf{x}$ & $\mathbf{x}$ & - & - & - & $\mathbf{x}$ & - & - & - & $\mathbf{x}$ & $\mathbf{x}$ & - & - & $\mathbf{x}$ & $\mathbf{x}$ & $\mathbf{x}$ & - & $\mathbf{x}$ \\
\hline Runs about & $\mathbf{x}$ & $\mathbf{x}$ & - & $\mathbf{x}$ & $\mathbf{x}$ & $\mathbf{x}$ & $\mathbf{x}$ & - & - & - & $\mathbf{x}$ & - & - & - & - & $\mathbf{x}$ & - & - & - & - & $\mathbf{x}$ & $\mathbf{x}$ & $\mathbf{x}$ & $\mathbf{x}$ & - \\
\hline \multicolumn{26}{|c|}{ WITH IMPULSIVITY } \\
\hline $\begin{array}{l}\text { Answering hastily / } \\
\text { hasty speech }\end{array}$ & $\mathbf{x}$ & - & $\mathbf{x}$ & $\mathbf{x}$ & $\mathbf{x}$ & - & - & $\mathbf{x}$ & - & $\mathbf{x}$ & $\mathbf{x}$ & $\mathbf{x}$ & $\mathbf{x}$ & $\mathbf{x}$ & - & $\mathbf{x}$ & $\mathbf{x}$ & & $\mathbf{x}$ & $\mathbf{x}$ & $\mathbf{x}$ & $\mathbf{x}$ & - & $\mathbf{x}$ & - \\
\hline Heedless & - & $\mathbf{x}$ & $\mathbf{x}$ & - & $\mathbf{x}$ & - & - & $\mathbf{x}$ & $\mathbf{x}$ & $\mathbf{x}$ & $\mathbf{x}$ & $\mathbf{x}$ & - & $\mathbf{x}$ & - & $\mathbf{x}$ & - & - & - & $\mathbf{x}$ & $\mathbf{x}$ & $\mathbf{x}$ & - & $\mathbf{x}$ & $\mathbf{x}$ \\
\hline Hurry + haste & $\mathbf{x}$ & - & - & $\mathbf{x}$ & $\mathbf{x}$ & - & $\mathbf{x}$ & $\mathbf{x}$ & $\mathbf{x}$ & $\mathbf{x}$ & $\mathbf{x}$ & $\mathbf{x}$ & $\mathbf{x}$ & $\mathbf{x}$ & $\mathbf{x}$ & $\mathbf{x}$ & $\mathbf{x}$ & $\mathbf{x}$ & $\mathbf{x}$ & $\mathbf{x}$ & $\mathbf{x}$ & $\mathbf{x}$ & $\mathbf{x}$ & $\mathbf{x}$ & - \\
\hline $\begin{array}{l}\text { Impulsive + } \\
\text { impetuous }\end{array}$ & $\mathbf{x}$ & - & $\mathbf{x}$ & $\mathbf{x}$ & - & - & - & $\mathbf{x}$ & $\mathbf{x}$ & $\mathbf{x}$ & - & $\mathbf{x}$ & - & $\mathbf{x}$ & $\mathbf{x}$ & $\mathbf{x}$ & - & $\mathbf{x}$ & - & $\mathbf{x}$ & - & $\mathbf{x}$ & - & - & $\mathbf{x}$ \\
\hline $\begin{array}{l}\text { Meddlesome + } \\
\text { importunate }\end{array}$ & $\mathbf{x}$ & - & - & - & - & - & $\mathbf{x}$ & $\mathbf{x}$ & - & - & $\mathbf{x}$ & - & - & $\mathbf{x}$ & - & $\mathbf{x}$ & - & - & - & $\mathbf{x}$ & $\mathbf{x}$ & - & - & $\mathbf{x}$ & - \\
\hline $\begin{array}{l}\text { Temerity + audacity + } \\
\text { courageous }\end{array}$ & $\mathbf{x}$ & $\mathbf{x}$ & - & - & $\mathbf{x}$ & - & - & - & - & $\mathbf{x}$ & $\mathbf{x}$ & $\mathbf{x}$ & - & $\mathbf{x}$ & - & - & $\mathbf{x}$ & $\mathbf{x}$ & - & $\mathbf{x}$ & - & $\mathbf{x}$ & - & $\mathbf{x}$ & - \\
\hline
\end{tabular}




\begin{tabular}{|c|c|c|c|c|c|c|c|c|c|c|c|c|c|c|c|c|c|c|c|c|c|c|c|c|c|}
\hline $\begin{array}{l}\text { Anger easily }+ \text { at } \\
\text { trifles }\end{array}$ & $\mathbf{x}$ & - & $\mathbf{x}$ & $\mathbf{x}$ & $\mathbf{x}$ & - & $\mathbf{x}$ & - & $\mathbf{x}$ & $\mathbf{x}$ & - & $\mathbf{x}$ & $\mathbf{x}$ & - & $\mathbf{x}$ & $\mathbf{x}$ & - & $\mathbf{x}$ & - & $\mathbf{x}$ & $\mathbf{x}$ & - & - & - & - \\
\hline Anger, rage, fury & $\mathbf{x}$ & $\mathbf{x}$ & $\mathbf{x}$ & $\mathbf{x}$ & $\mathbf{x}$ & $\mathbf{x}$ & $\mathbf{x}$ & $\mathbf{x}$ & $\mathbf{x}$ & $\mathbf{x}$ & $\mathbf{x}$ & $\mathbf{x}$ & $\mathbf{x}$ & $\mathbf{x}$ & $\mathbf{x}$ & $\mathbf{x}$ & $\mathbf{x}$ & $\mathbf{x}$ & $\mathbf{x}$ & $\mathbf{x}$ & $\mathbf{x}$ & $\mathbf{x}$ & $\mathbf{x}$ & $\mathbf{x}$ & $\mathbf{x}$ \\
\hline Biting & $\mathbf{x}$ & - & - & - & $\mathbf{x}$ & $\mathbf{x}$ & $\mathbf{x}$ & - & - & - & $\mathbf{x}$ & $\mathbf{x}$ & - & - & - & - & $\mathbf{x}$ & $\mathbf{x}$ & - & - & $\mathbf{x}$ & - & - & $\mathbf{x}$ & - \\
\hline Biting nails & $\mathbf{x}$ & - & - & $\mathbf{x}$ & - & - & $\mathbf{x}$ & $\mathbf{x}$ & - & - & $\mathbf{x}$ & $\mathbf{x}$ & $\mathbf{x}$ & - & $\mathbf{x}$ & - & & $\mathbf{x}$ & - & - & $\mathbf{x}$ & $\mathbf{x}$ & - & $\mathbf{x}$ & - \\
\hline Corrupt + dishonest & - & - & - & $\mathbf{x}$ & - & - & $\mathbf{x}$ & - & - & - & - & $\mathbf{x}$ & $\mathbf{x}$ & - & - & - & - & - & - & - & - & $\mathbf{x}$ & - & - & - \\
\hline Cruelty & - & - & $\mathbf{x}$ & $\mathbf{x}$ & $\mathbf{x}$ & - & $\mathbf{x}$ & - & $\mathbf{x}$ & $\mathbf{x}$ & $\mathbf{x}$ & $\mathbf{x}$ & $\mathbf{x}$ & - & $\mathbf{x}$ & $\mathbf{x}$ & $\mathbf{x}$ & - & - & $\mathbf{x}$ & $\mathbf{x}$ & - & $\mathbf{x}$ & $\mathbf{x}$ & - \\
\hline Escape, attempts & $\mathbf{x}$ & $\mathbf{x}$ & - & $\mathbf{x}$ & $\mathbf{x}$ & - & - & $\mathbf{x}$ & $\mathbf{x}$ & - & $\mathbf{x}$ & $\mathbf{x}$ & $\mathbf{x}$ & $\mathbf{x}$ & - & $\mathbf{x}$ & $\mathbf{x}$ & $\mathbf{x}$ & $\mathbf{x}$ & - & $\mathbf{x}$ & - & $\mathbf{x}$ & $\mathbf{x}$ & - \\
\hline Hiding himself & - & - & - & $\mathbf{x}$ & $\mathbf{x}$ & - & - & - & - & - & $\mathbf{x}$ & $\mathbf{x}$ & - & - & - & - & - & & - & $\mathbf{x}$ & $\mathbf{x}$ & - & $\mathbf{x}$ & - & - \\
\hline Lascivious & $\mathbf{x}$ & - & $\mathbf{x}$ & $\mathbf{x}$ & $\mathbf{x}$ & $\mathbf{x}$ & $\mathbf{x}$ & $\mathbf{x}$ & - & $\mathbf{x}$ & $\mathbf{x}$ & $\mathbf{x}$ & $\mathbf{x}$ & $\mathbf{x}$ & - & $\mathbf{x}$ & $\mathbf{x}$ & $\mathbf{x}$ & $\mathbf{x}$ & - & - & $\mathbf{x}$ & - & $\mathbf{x}$ & $\mathbf{x}$ \\
\hline $\begin{array}{l}\text { Liar }+ \text { deceitful }+ \\
\text { untruthful }+ \text { false }+ \\
\text { perfidious }\end{array}$ & - & $\mathbf{x}$ & $\mathbf{x}$ & $\mathbf{x}$ & $\mathbf{x}$ & $\mathbf{x}$ & $\mathbf{x}$ & $\mathbf{x}$ & - & - & $\mathbf{x}$ & $\mathbf{x}$ & $\mathbf{x}$ & $\mathbf{x}$ & $\mathbf{x}$ & $\mathbf{x}$ & $\mathbf{x}$ & $\mathbf{x}$ & - & $\mathbf{x}$ & - & $\mathbf{x}$ & $\mathbf{x}$ & $\mathbf{x}$ & - \\
\hline Mischievous & - & $\mathbf{x}$ & $\mathbf{x}$ & $\mathbf{x}$ & - & $\mathbf{x}$ & $\mathbf{x}$ & - & $\mathbf{x}$ & - & $\mathbf{x}$ & $\mathbf{x}$ & - & $\mathbf{x}$ & $\mathbf{x}$ & - & - & - & - & - & $\mathbf{x}$ & - & $\mathbf{x}$ & $\mathbf{x}$ & - \\
\hline $\begin{array}{l}\text { Moral feeling, wants } \\
\text { of }+ \text { no sense of duty }\end{array}$ & - & - & $\mathbf{x}$ & $\mathbf{x}$ & $\mathbf{x}$ & $\mathbf{x}$ & - & - & $\mathbf{x}$ & $\mathbf{x}$ & $\mathbf{x}$ & - & - & - & $\mathbf{x}$ & $\mathbf{x}$ & $\mathbf{x}$ & - & - & - & $\mathbf{x}$ & - & $\mathbf{x}$ & $\mathbf{x}$ & - \\
\hline Perversity & - & - & $\mathbf{x}$ & - & - & - & - & - & - & - & - & - & - & - & - & - & - & - & - & - & - & - & - & - & - \\
\hline Pulling hair & - & - & - & $\mathbf{x}$ & $\mathbf{x}$ & - & - & - & - & - & - & $\mathbf{x}$ & - & $\mathbf{x}$ & - & - & - & - & - & - & $\mathbf{x}$ & - & $\mathbf{x}$ & - & - \\
\hline Set things on fire & - & - & - & - & $\mathbf{x}$ & - & $\mathbf{x}$ & - & - & $\mathbf{x}$ & $\mathbf{x}$ & - & - & - & - & - & - & $\mathbf{x}$ & - & $\mathbf{x}$ & $\mathbf{x}$ & - & - & - & - \\
\hline Shameless+ obscene & - & - & $\mathbf{x}$ & - & $\mathbf{x}$ & $\mathbf{x}$ & $\mathbf{x}$ & $\mathbf{x}$ & - & - & $\mathbf{x}$ & $\mathbf{x}$ & $\mathbf{x}$ & $\mathbf{x}$ & - & $\mathbf{x}$ & $\mathbf{x}$ & $\mathbf{x}$ & - & $\mathbf{x}$ & $\mathbf{x}$ & $\mathbf{x}$ & $\mathbf{x}$ & $\mathbf{x}$ & \\
\hline Shrieking, children & $\mathbf{x}$ & - & $\mathbf{x}$ & - & $\mathbf{x}$ & - & $\mathbf{x}$ & - & $\mathbf{x}$ & - & - & - & $\mathbf{x}$ & - & - & $\mathbf{x}$ & - & $\mathbf{x}$ & - & - & $\mathbf{x}$ & - & - & - & - \\
\hline Spiting & - & - & - & - & $\mathbf{x}$ & $\mathbf{x}$ & $\mathbf{x}$ & - & - & - & - & - & $\mathbf{x}$ & $\mathbf{x}$ & - & $\mathbf{x}$ & $\mathbf{x}$ & - & - & - & - & $\mathbf{x}$ & - & $\mathbf{x}$ & - \\
\hline Stealing & - & - & - & $\mathbf{x}$ & $\mathbf{x}$ & & $\mathbf{x}$ & $\mathbf{x}$ & - & - & - & - & $\mathbf{x}$ & - & - & $\mathbf{x}$ & - & - & - & $\mathbf{x}$ & $\mathbf{x}$ & $\mathbf{x}$ & $\mathbf{x}$ & - & - \\
\hline $\begin{array}{l}\text { Striking + attack + } \\
\text { fight + kicking }\end{array}$ & $\mathbf{x}$ & $\mathbf{x}$ & - & - & $\mathbf{x}$ & - & - & - & $\mathbf{x}$ & - & $\mathbf{x}$ & - & - & $\mathbf{x}$ & - & $\mathbf{x}$ & - & - & $\mathbf{x}$ & $\mathbf{x}$ & $\mathbf{x}$ & $\mathbf{x}$ & $\mathbf{x}$ & $\mathbf{x}$ & - \\
\hline $\begin{array}{l}\text { Tearing, tormenting, } \\
\text { biting, striking } \\
\text { himself, knocking his } \\
\text { head }\end{array}$ & $\mathbf{x}$ & $\mathbf{x}$ & - & $\mathbf{x}$ & $\mathbf{x}$ & - & - & - & - & - & $\mathbf{x}$ & - & - & - & - & - & $\mathbf{x}$ & $\mathbf{x}$ & $\mathbf{x}$ & - & $\mathbf{x}$ & $\mathbf{x}$ & $\mathbf{x}$ & $\mathbf{x}$ & - \\
\hline Threatening & - & $\mathbf{x}$ & - & - & - & - & - & - & - & $\mathbf{x}$ & - & - & - & - & - & - & - & - & - & - & $\mathbf{x}$ & - & $\mathbf{x}$ & - & - \\
\hline $\begin{array}{l}\text { Throwing, tearing, } \\
\text { breaking things }+ \text { des- }\end{array}$ & $\mathbf{x}$ & $\mathbf{x}$ & $\mathbf{x}$ & $\mathbf{x}$ & $\mathbf{x}$ & $\mathbf{x}$ & $\mathbf{x}$ & $\mathbf{x}$ & $\mathbf{x}$ & $\mathbf{x}$ & $\mathbf{x}$ & $\mathbf{x}$ & $\mathbf{x}$ & $\mathbf{x}$ & - & $\mathbf{x}$ & $\mathbf{x}$ & $\mathbf{x}$ & $\mathbf{x}$ & $\mathbf{x}$ & $\mathbf{x}$ & $\mathbf{x}$ & $\mathbf{x}$ & $\mathbf{x}$ & - \\
\hline
\end{tabular}




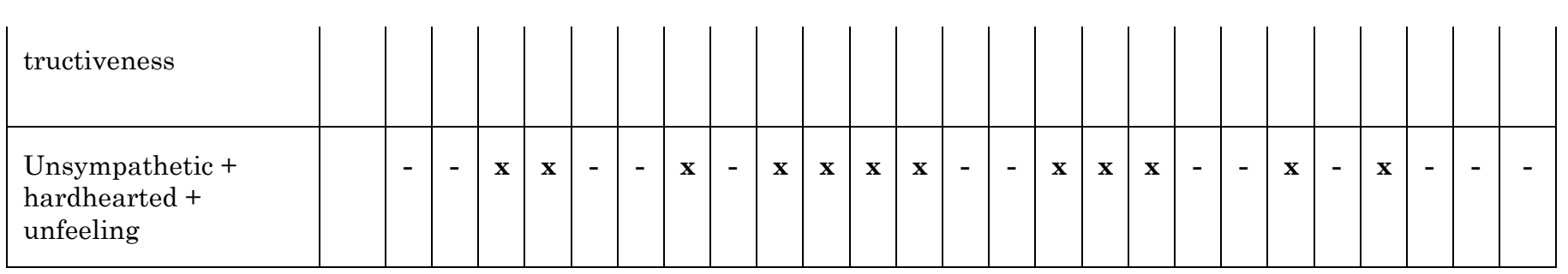

\begin{tabular}{|c|c|c|c|c|c|c|c|c|c|c|c|c|c|c|c|c|c|c|c|c|c|c|c|c|c|}
\hline \multicolumn{26}{|c|}{ OPPOSITIONAL DEFIANT DISORDER (IDC - 313.81) } \\
\hline Abusive + cursing & $\mathbf{x}$ & - & $\mathbf{x}$ & $\mathbf{x}$ & $\mathbf{x}$ & $\mathbf{x}$ & $\mathbf{x}$ & $\mathbf{x}$ & $\mathbf{x}$ & $\mathbf{x}$ & $\mathbf{x}$ & $\mathbf{x}$ & $\mathbf{x}$ & $\mathbf{x}$ & $\mathbf{x}$ & $\mathbf{x}$ & $\mathbf{x}$ & $\mathbf{x}$ & $\mathbf{x}$ & $\mathbf{x}$ & $\mathbf{x}$ & - & $\mathbf{x}$ & $\mathbf{x}$ & - \\
\hline $\begin{array}{l}\text { Contradiction, } \\
\text { intolerant }+ \text { anger } \\
\text { from contradiction }\end{array}$ & $\mathbf{x}$ & - & $\mathbf{x}$ & $\mathbf{x}$ & $\mathbf{x}$ & - & $\mathbf{x}$ & - & $\mathbf{x}$ & $\mathbf{x}$ & $\mathbf{x}$ & $\mathbf{x}$ & $\mathbf{x}$ & $\mathbf{x}$ & $\mathbf{x}$ & $\mathbf{x}$ & $\mathbf{x}$ & $\mathbf{x}$ & - & $\mathbf{x}$ & $\mathbf{x}$ & - & $\mathbf{x}$ & $\mathbf{x}$ & - \\
\hline $\begin{array}{l}\text { Contradiction, } \\
\text { weeping from }\end{array}$ & - & - & - & - & - & - & - & - & - & - & $\mathbf{x}$ & - & - & - & - & $\mathbf{x}$ & - & - & - & - & $\mathbf{x}$ & - & - & - & - \\
\hline Defiant + inciting & $\mathbf{x}$ & - & $\mathbf{x}$ & - & $\mathbf{x}$ & $\mathbf{x}$ & - & $\mathbf{x}$ & - & - & $\mathbf{x}$ & - & $\mathbf{x}$ & - & - & $\mathbf{x}$ & - & - & - & - & - & $\mathbf{x}$ & - & - & - \\
\hline $\begin{array}{l}\text { Disobedience + } \\
\text { contrary }\end{array}$ & $\mathbf{x}$ & - & $\mathbf{x}$ & $\mathbf{x}$ & $\mathbf{x}$ & $\mathbf{x}$ & $\mathbf{x}$ & $\mathbf{x}$ & $\mathbf{x}$ & $\mathbf{x}$ & - & $\mathbf{x}$ & $\mathbf{x}$ & $\mathbf{x}$ & $\mathbf{x}$ & $\mathbf{x}$ & - & $\mathbf{x}$ & $\mathbf{x}$ & $\mathbf{x}$ & - & $\mathbf{x}$ & $\mathbf{x}$ & - & - \\
\hline Impatient & $\mathbf{x}$ & - & $\mathbf{x}$ & $\mathbf{x}$ & $\mathbf{x}$ & $\mathbf{x}$ & $\mathbf{x}$ & - & $\mathbf{x}$ & $\mathbf{x}$ & $\mathbf{x}$ & $\mathbf{x}$ & $\mathbf{x}$ & $\mathbf{x}$ & $\mathbf{x}$ & $\mathbf{x}$ & $\mathbf{x}$ & $\mathbf{x}$ & - & $\mathbf{x}$ & - & $\mathbf{x}$ & $\mathbf{x}$ & - & $\mathbf{x}$ \\
\hline $\begin{array}{l}\text { Impertinence }+ \\
\text { insolence }\end{array}$ & - & - & $\mathbf{x}$ & $\mathbf{x}$ & $\mathbf{x}$ & $\mathbf{x}$ & $\mathbf{x}$ & - & $\mathbf{x}$ & - & $\mathbf{x}$ & $\mathbf{x}$ & $\mathbf{x}$ & - & $\mathbf{x}$ & $\mathbf{x}$ & - & $\mathbf{x}$ & - & $\mathbf{x}$ & $\mathbf{x}$ & $\mathbf{x}$ & $\mathbf{x}$ & $\mathbf{x}$ & - \\
\hline Malicious + hatred & $\mathbf{x}$ & $\mathbf{x}$ & $\mathbf{x}$ & $\mathbf{x}$ & $\mathbf{x}$ & - & $\mathbf{x}$ & $\mathbf{x}$ & $\mathbf{x}$ & $\mathbf{x}$ & $\mathbf{x}$ & $\mathbf{x}$ & $\mathbf{x}$ & $\mathbf{x}$ & $\mathbf{x}$ & $\mathbf{x}$ & $\mathbf{x}$ & $\mathbf{x}$ & - & $\mathbf{x}$ & $\mathbf{x}$ & $\mathbf{x}$ & $\mathbf{x}$ & $\mathbf{x}$ & $\mathbf{x}$ \\
\hline Mocking + jesting & - & $\mathbf{x}$ & - & $\mathbf{x}$ & $\mathbf{x}$ & - & $\mathbf{x}$ & $\mathbf{x}$ & - & - & $\mathbf{x}$ & $\mathbf{x}$ & $\mathbf{x}$ & $\mathbf{x}$ & - & $\mathbf{x}$ & - & - & - & $\mathbf{x}$ & $\mathbf{x}$ & - & $\mathbf{x}$ & - & - \\
\hline $\begin{array}{l}\text { Offended, easily + } \\
\text { irritability about } \\
\text { trifles }\end{array}$ & $\mathbf{x}$ & $\mathbf{x}$ & $\mathbf{x}$ & $\mathbf{x}$ & $\mathbf{x}$ & $\mathbf{x}$ & $\mathbf{x}$ & $\mathbf{x}$ & $\mathbf{x}$ & $\mathbf{x}$ & $\mathbf{x}$ & $\mathbf{x}$ & $\mathbf{x}$ & $\mathbf{x}$ & $\mathbf{x}$ & $\mathbf{x}$ & - & $\mathbf{x}$ & - & $\mathbf{x}$ & $\mathbf{x}$ & $\mathbf{x}$ & - & $\mathbf{x}$ & $\mathbf{x}$ \\
\hline Quarrelsome & $\mathbf{x}$ & $\mathbf{x}$ & $\mathbf{x}$ & $\mathbf{x}$ & $\mathbf{x}$ & $\mathbf{x}$ & $\mathbf{x}$ & $\mathbf{x}$ & $\mathbf{x}$ & $\mathbf{x}$ & $\mathbf{x}$ & $\mathbf{x}$ & $\mathbf{x}$ & $\mathbf{x}$ & $\mathbf{x}$ & $\mathbf{x}$ & $\mathbf{x}$ & $\mathbf{x}$ & $\mathbf{x}$ & $\mathbf{x}$ & $\mathbf{x}$ & $\mathbf{x}$ & $\mathbf{x}$ & $\mathbf{x}$ & $\mathbf{x}$ \\
\hline Teasing + wearisome & $\mathbf{x}$ & - & $\mathbf{x}$ & $\mathbf{x}$ & $\mathbf{x}$ & - & $\mathbf{x}$ & $\mathbf{x}$ & $\mathbf{x}$ & $\mathbf{x}$ & - & $\mathbf{x}$ & $\mathbf{x}$ & $\mathbf{x}$ & - & $\mathbf{x}$ & - & $\mathbf{x}$ & - & $\mathbf{x}$ & - & $\mathbf{x}$ & - & - & $\mathbf{x}$ \\
\hline Wildness + rage & $\mathbf{x}$ & - & - & - & $\mathbf{x}$ & $\mathbf{x}$ & $\mathbf{x}$ & - & - & - & $\mathbf{x}$ & - & - & - & - & - & $\mathbf{x}$ & $\mathbf{x}$ & - & - & $\mathbf{x}$ & - & - & $\mathbf{x}$ & - \\
\hline
\end{tabular}




\begin{tabular}{|c|c|c|c|c|c|c|c|c|c|c|c|c|c|c|c|c|c|c|c|c|c|c|c|c|c|}
\hline Involuntary stool & - & - & - & $\mathbf{x}$ & - & $\mathbf{x}$ & $\mathbf{x}$ & $\mathbf{x}$ & - & - & $\mathbf{x}$ & $\mathbf{x}$ & - & $\mathbf{x}$ & - & $\mathbf{x}$ & $\mathbf{x}$ & $\mathbf{x}$ & $\mathbf{x}$ & $\mathbf{x}$ & $\mathbf{x}$ & $\mathbf{x}$ & $\mathbf{x}$ & $\mathbf{x}$ & $\mathbf{x}$ \\
\hline $\begin{array}{l}\text { Involuntary urination } \\
\text { at night }\end{array}$ & $\mathbf{x}$ & - & $\mathbf{x}$ & $\mathbf{x}$ & $\mathbf{x}$ & - & $\mathbf{x}$ & $\mathbf{x}$ & $\mathbf{x}$ & $\mathbf{x}$ & $\mathbf{x}$ & - & $\mathbf{x}$ & $\mathbf{x}$ & $\mathbf{x}$ & $\mathbf{x}$ & $\mathbf{x}$ & $\mathbf{x}$ & & $\mathbf{x}$ & $\mathbf{x}$ & $\mathbf{x}$ & - & $\mathbf{x}$ & $\mathbf{x}$ \\
\hline Restless during sleep & $\mathbf{x}$ & $\mathbf{x}$ & $\mathbf{x}$ & $\mathbf{x}$ & $\mathbf{x}$ & $\mathbf{x}$ & $\mathbf{x}$ & $\mathbf{x}$ & $\mathbf{x}$ & $\mathbf{x}$ & $\mathbf{x}$ & $\mathbf{x}$ & - & $\mathbf{x}$ & $\mathbf{x}$ & $\mathbf{x}$ & $\mathbf{x}$ & $\mathbf{x}$ & $\mathbf{x}$ & $\mathbf{x}$ & $\mathbf{x}$ & $\mathbf{x}$ & $\mathbf{x}$ & $\mathbf{x}$ & $\mathbf{x}$ \\
\hline Handles genitals & $\mathbf{x}$ & - & - & - & $\mathbf{x}$ & $\mathbf{x}$ & - & - & - & - & $\mathbf{x}$ & - & - & $\mathbf{x}$ & - & - & - & - & - & - & $\mathbf{x}$ & - & - & - & $\mathbf{x}$ \\
\hline $\begin{array}{l}\text { Masturbation, } \\
\text { disposition to }\end{array}$ & - & - & $\mathbf{x}$ & - & $\mathbf{x}$ & $\mathbf{x}$ & $\mathbf{x}$ & $\mathbf{x}$ & - & - & $\mathbf{x}$ & $\mathbf{x}$ & $\mathbf{x}$ & $\mathbf{x}$ & $\mathbf{x}$ & $\mathbf{x}$ & $\mathbf{x}$ & $\mathbf{x}$ & - & $\mathbf{x}$ & $\mathbf{x}$ & $\mathbf{x}$ & $\mathbf{x}$ & - & $\mathbf{x}$ \\
\hline Naked, wishes stay & - & - & - & - & $\mathbf{x}$ & $\mathbf{x}$ & - & - & $\mathbf{x}$ & - & $\mathbf{x}$ & - & - & $\mathbf{x}$ & - & - & - & $\mathbf{x}$ & - & - & $\mathbf{x}$ & - & $\mathbf{x}$ & $\mathbf{x}$ & - \\
\hline Nightmares & $\mathbf{x}$ & $\mathbf{x}$ & $\mathbf{x}$ & $\mathbf{x}$ & $\mathbf{x}$ & $\mathbf{x}$ & $\mathbf{x}$ & $\mathbf{x}$ & $\mathbf{x}$ & $\mathbf{x}$ & $\mathbf{x}$ & $\mathbf{x}$ & $\mathbf{x}$ & $\mathbf{x}$ & $\mathbf{x}$ & $\mathbf{x}$ & $\mathbf{x}$ & $\mathbf{x}$ & - & $\mathbf{x}$ & $\mathbf{x}$ & $\mathbf{x}$ & $\mathbf{x}$ & $\mathbf{x}$ & $\mathbf{x}$ \\
\hline $\begin{array}{l}\text { Grinding teeth during } \\
\text { sleep }\end{array}$ & $\mathbf{x}$ & $\mathbf{x}$ & - & $\mathbf{x}$ & $\mathbf{x}$ & - & $\mathbf{x}$ & $\mathbf{x}$ & - & - & $\mathbf{x}$ & - & - & $\mathbf{x}$ & - & - & - & - & $\mathbf{x}$ & - & $\mathbf{x}$ & $\mathbf{x}$ & - & $\mathbf{x}$ & $\mathbf{x}$ \\
\hline Fear at night & $\mathbf{x}$ & - & - & - & - & - & $\mathbf{x}$ & - & $\mathbf{x}$ & - & - & - & $\mathbf{x}$ & - & - & - & $\mathbf{x}$ & $\mathbf{x}$ & - & - & $\mathbf{x}$ & - & - & - & - \\
\hline Grimaces & - & $\mathbf{x}$ & - & $\mathbf{x}$ & $\mathbf{x}$ & - & $\mathbf{x}$ & $\mathbf{x}$ & - & - & $\mathbf{x}$ & $\mathbf{x}$ & $\mathbf{x}$ & $\mathbf{x}$ & - & $\mathbf{x}$ & $\mathbf{x}$ & $\mathbf{x}$ & $\mathbf{x}$ & - & $\mathbf{x}$ & $\mathbf{x}$ & $\mathbf{x}$ & $\mathbf{x}$ & $\mathbf{x}$ \\
\hline
\end{tabular}

Table 2 - Compared Materia Medica for Patterns of Violent Behavior

$\begin{array}{ll}\text { Aconitum } & \begin{array}{l}\text { Reading and attention disorders, with hyperactivity and impulsiveness. Fear of death } \\ \text { dominates everything and generates a basic state of anxiety, alertness, sensory and } \\ \text { emotional hypersensitivity. Panic sudden crisis, anxiety with high nervous excitability, } \\ \text { makes everything in a hurry, changes position constantly; fear to leave or to stay in a } \\ \text { room with many persons. "World is a dangerous place where only disgraceful things } \\ \text { happen takes place" (Masi Elizalde). Fear to be injured, prudence, cowardice: his face } \\ \text { express fear, predicts the day of his death, anything frightens - disturbed by scare and } \\ \text { fear; the child is mischievous and afraid of darkness - night terror. In another extreme is } \\ \text { audacious and courageous, impetuous, quarrelsome, disobedient; angry, throws and } \\ \text { breaks things, "self and hetero aggression", bites his own nails. Stammers, bites, contrary, } \\ \text { oppositional defiant. Enuresis. } \\ \text { aggravates from wind. }\end{array}\end{array}$




\begin{tabular}{|c|c|}
\hline $\begin{array}{l}\text { Agaricus } \\
\text { muscarius }\end{array}$ & $\begin{array}{l}\text { Reading, writing and language disorders; ADHD with impulsiveness. Great instability, } \\
\text { irritability and depression alternate. Choleric with himself and hetero-aggression - } \\
\text { threatening. He values especially physical force; does not accept authority, takes it as } \\
\text { arbitrariness; a child that presents himself as an adult (Masi Elizalde). Cold, bloodthirsty } \\
\text { behavior, worrier spirit; likes to be close to fire. Involuntary movements of the muscles, } \\
\text { tremors, choreic movements - nervous tics. They like to dance, extreme need of dancing; } \\
\text { megalomania, exaggerated boldness, everything is exaggerated in him. Crises of } \\
\text { spasmodic crying, depressive state, and sorrow by the most trivial thing: joy is } \\
\text { transformed into suffering. Delay to talk and to walk (as in Calc.), due to slow brain } \\
\text { development; face has a foolish expression, like intoxicated; dumbness and physical and } \\
\text { mental lack of coordination; mind and sensorial look paralyzed. Aversion to be touched, } \\
\text { and to mental and physical work. Sensation as if ice needles in the skin, aching sensation } \\
\text { as stung. Very sensitive to coldness, worse from cold air. }\end{array}$ \\
\hline $\begin{array}{l}\text { Anacardium } \\
\text { orientale }\end{array}$ & $\begin{array}{l}\text { Internal conflict is the central subject: division of will, tremendous indecision between } \\
\text { good and evil - two opposite wills, one allows and the other prohibits. They are ambivalent, } \\
\text { personality with two distinct sides: oscillates between aggressiveness and dependence; } \\
\text { feels aggressive and passive at the same time, like strong and weak. Cannot make } \\
\text { decisions concerning values and things to be considered; indecision while choosing } \\
\text { between good and bad (Masi Elizalde). Religious delirium, with worry to save his soul; } \\
\text { cruelty with impulse to kill, little concern with others; impulse to attack with violent } \\
\text { sexual language. Olfactory and hearing hallucinations. He is easily offended. Infants and } \\
\text { temperamental youths or psychopaths, socially maladjusted, with unscrupulous violent, } \\
\text { contradictory and immediate demands; cruelty towards animals, sadism, inhuman, } \\
\text { merciless, malevolent, insults with swearwords - perversity. At the same time, } \\
\text { abandoned, despaired, imploring; nervous individuals, deadened, weak, generally thin, } \\
\text { chilly, a lot sensible to draughts. It is important for him to show his importance for the } \\
\text { others and the world; suffers from a deep complex of inferiority, with sensation of isolation } \\
\text { and of having been abandoned. Feels strong internal rage due to his feeling of inferiority } \\
\text { and dissatisfaction, leading him to aggressive and cruel behavior when he feels insecure, } \\
\text { only attacks when in company or when his victims cannot defend themselves. Thus, he } \\
\text { expresses his sadistic and cruel side with feelings of superiority and pleasure. At the } \\
\text { mirror, he blames everybody, except himself. An important characteristic is the great } \\
\text { relief after eating; however, the symptoms come back and increase in intensity so that the } \\
\text { patient is forced to eat again, looking for relief. }\end{array}$ \\
\hline $\begin{array}{l}\text { Arsenicum } \\
\text { album }\end{array}$ & $\begin{array}{l}\text { Anxious, fearful, restless, irritable, sensitive, easily angry. Changes constantly from place } \\
\text { to place, due to early morning anxiety attacks, he leaves the bed in the middle of the night. } \\
\text { Grasps people to feel secure. Fear of being alone, of darkness; wakes up terrified and } \\
\text { jumps out the bed, searches for someone to speak with, hiding under his bed. Delusion } \\
\text { with bugs in his bed and thieves in the room, fear of thieves. Self-attack, beats his own } \\
\text { head against the wall, attacks others. Wicked, liar, jealous, perverse, steals things. ADD } \\
\text { with hyperactivity, behavior and oppositional defiant. Meticulous, collector, stingy. He } \\
\text { wants everything planned and ritualized. Fear of illness, emotionally impressed. } \\
\text { Language and speech disorders, stammer, nervous tics, enuresis and encopresis, grinds } \\
\text { the teeth. Hypersensitive to cold, chilly with desire of cold beverages. Intolerant to smells } \\
\text { and noises, perturbations by noises. Skin eruption alternating with asthma, or asthma } \\
\text { and diarrhea. Theme of responsibility regarding order and law (Masi Elizalde). }\end{array}$ \\
\hline
\end{tabular}




\begin{tabular}{|c|c|}
\hline Belladona & $\begin{array}{l}\text { Lively persons and amused when are well, but violent and frequently delirious when sick; } \\
\text { sensitive to draughts, especially on uncovering head; aggravate by cutting hair. } \\
\text { Unsuitable for mathematics, language disorder. ADHD with impulsiveness, oppositional } \\
\text { defiant. Fear of imaginary things: imagines ghosts, horrible faces and several bugs; black } \\
\text { animals, dogs, wolves. Tendency to bite, spit, beat and tear things; attacks of laughter and } \\
\text { grinds the teeth; tries to run away from home. Desire to set on fire and steal. Abrupt and } \\
\text { intense acute illness, pains come suddenly, lasting indefinite time and cease suddenly. } \\
\text { Affluence of blood to head and face, with blush and heat, looking wild, mydriasis, arteries } \\
\text { pulsation. Enuresis, tics and grimaces. Aggravates by reproaches. }\end{array}$ \\
\hline Bufo rana & $\begin{array}{l}\text { It acts mainly on CNS and skin. Indicated for children with weak mind (idiocy or } \\
\text { oligofrenia), childish behavior, increased desire for masturbation, hands are always } \\
\text { touching genitals. Desires to be alone to practice masturbation, but fears to stay alone. } \\
\text { Irritable, with tendency to bite, bites his own tongue. Choleric behavior, jumps off the bed } \\
\text { and runs like a lunatic. Epilepsy, with convulsions that begin during sleep. Laughs like } \\
\text { a fool, cries and laughs easily. Does not tolerate music or bright objects. Tendency to } \\
\text { injure his own fingers. Desire of alcoholic beverages. Lack of control of his sexuality, } \\
\text { perverse practices and addictions (Kent). Twists hands in anxiety, as something terrible is } \\
\text { going to happen. }\end{array}$ \\
\hline $\begin{array}{l}\text { Calcarea } \\
\text { carbonica }\end{array}$ & $\begin{array}{l}\text { Fear is its main characteristic: of suffering, of hunger, of illness, of sad or horrible stories. } \\
\text { Prudent, does not take risks. He needs emotional and physical stability in order to feel } \\
\text { secure. When destabilized, agitates himself in a disorganized way, goes into raptures, } \\
\text { bites, spites at people, ready to kill. Emotional, fear of everything, full of fantasies. } \\
\text { Difficulty in psycho-motor development and teething. Ashen, cold, soft, flaccid, lazy, wet. } \\
\text { Slow, heavy, tendency to obesity or weight loss. Aggravates by reproaches, fright, fear, } \\
\text { anticipation, rudeness and violence. ADD. Theme of secureness about his health and } \\
\text { future - lack of protection (Masi Elizalde). }\end{array}$ \\
\hline Causticum & $\begin{array}{l}\text { Compassionate towards others' suffering feels himself in the place of others because the } \\
\text { same can happen to him. Total identification with the others' situation, revolutionary due } \\
\text { to compassion. Theme of lack of protection (Masi Elizalde). Melancholic humor, } \\
\text { despaired, by worry, sorrow, full of fears - stays paralyzed; with crying, for the least cause. } \\
\text { Prudent, avoids risks. Perturbations by affliction or sorrow of long duration, by loss of } \\
\text { sleep, nocturnal vigil, sudden emotions, fear, fright, joy, rage. Unable to stay quiet, needs } \\
\text { to move constantly, but the movement does not relieve him. Unsuitable for mathematics, } \\
\text { disturb of language, ADHD with impulsiveness. May become defiant, liar, anarchist, } \\
\text { oppositional, with tendency to steal. Enuresis, encopresis and nervous tics. Weight loss } \\
\text { and ponderal stagnation, despite good appetite. Tendency to warts, mainly around nails. }\end{array}$ \\
\hline
\end{tabular}




\begin{tabular}{|c|c|}
\hline $\begin{array}{l}\text { Matricaria } \\
\text { chamomilla }\end{array}$ & $\begin{array}{l}\text { Hypersensitiveness of the nervous system, mainly regarding noises, pain and people; bears } \\
\text { nobody nearby; ill humor, dislikes being talked to, aversion to conversation, answers } \\
\text { roughly ; irascible, he is never completely calm and in peace; ameliorates by movement } \\
\text { and has to be distracted all the time, irritable, throws things, hauls the hair of the others, } \\
\text { does not want to be touched, kicks; angry, quarrelsome, oppositional defiant, impatient, } \\
\text { wants many things and becomes furious when something that he wants is not given to } \\
\text { him, but when he gets it, rejects it; he is never satisfied; is easily bored with things that } \\
\text { were desired, soon wants another one, is never satisfied with what is doing; malevolent, } \\
\text { ailments from anger; he gets worse along day till night, when becomes unbearable; sleepy, } \\
\text { but sleeplessness. Uncontrollable children, stops breathing or starts a convulsion when in } \\
\text { anger, beats his head against the wall. The face is half red, half white in anger or fever. } \\
\text { Difficult teething. Fear of wind. Disturbs and difficulties to read, write and speak. ADHD } \\
\text { with impulsiveness. Nocturnal terror and enuresis. He cannot fulfill any physiological } \\
\text { function without suffering; feeling of being unfairly treated (Masi Elizalde). }\end{array}$ \\
\hline $\begin{array}{l}\text { Hepar } \\
\text { sulphuris } \\
\text { calcareum }\end{array}$ & $\begin{array}{l}\text { Physical and mental hypersensitiveness (to touch, to pain - faints from pain); anything } \\
\text { irritates him, anger at trifles; speaks and drinks in a hurry, hypochondriac, causeless } \\
\text { anxiety. Defiant, angry, threatens with knives, oppositional. Pyromaniac, extremely } \\
\text { violent, destructive; cruelty with sadism and satisfaction in front of other's suffering, but } \\
\text { is kind to animals - Purity is his theme (Masi Elizalde). Problems of speech, stammering, } \\
\text { and enuresis. ADD with impulsiveness, perverse impulses. Sensitive to cold air, needs to } \\
\text { cover his face even in hot climate; dislikes to be undressed. Even a small wound causes } \\
\text { suppuration. Ferocious anger and irrational impulsiveness make him a cold blooded } \\
\text { murderer that kills without remorse, even among those cheerful and friendly ones; feels as } \\
\text { if could kill someone with pleasure (Hering). }\end{array}$ \\
\hline $\begin{array}{l}\text { Hyosciamus } \\
\text { niger }\end{array}$ & $\begin{array}{l}\text { Irritable, nervous, hysterical, jealous (its basic characteristic). Fright or intestinal worms } \\
\text { provoke convulsions. Agitation, jumps off his bed, tries to escape; tells stories about } \\
\text { imaginary deeds. Fear of being alone, of being poisoned, of being bitten, of being sold, of } \\
\text { taking away what has been given to him, distrustful of some plots. Harmful effects of non } \\
\text { corresponded love, with jealous and anger; incoherent talk, tendency to laugh at } \\
\text { everything, dizzy laughter. Lascivious mania: impudently does not want to be covered, } \\
\text { strips clothes, exposes his body; sings obscene songs; goes naked to bed and exposes his } \\
\text { genitals, handles genitals. Disturbs of speech, stammering. ADHD with impulsiveness, } \\
\text { behavior disorder, oppositional defiant, savagery, ferocity. He bites, breaks everything, } \\
\text { injures himself on purpose. Enuresis, encopresis, tics, makes grimaces, acts as a clown, } \\
\text { ridiculous attitudes. Acute diseases with delirium and pale face. }\end{array}$ \\
\hline $\begin{array}{l}\text { Lachesis } \\
\text { muta }\end{array}$ & $\begin{array}{l}\text { Jealous, envious, excessive reactions: bites, injures others, wants to be the center of } \\
\text { attention. Distrustful, resentful and vindictive, liar and manipulator. Chatters, intense } \\
\text { loquacity changing from one theme to another. Disposition to drink alcoholic beverages } \\
\text { and indolence, cheerlessness, frequent masturbation. Theme of love and admiration, } \\
\text { truth and lie (Masi Elizalde). He feels abandoned, impressionable. Intuitive, precocious } \\
\text { and lively children. High sensitiveness to touch on his throat, stomach, abdomen; does not } \\
\text { bear tight clothes on throat or abdomen, they cause uneasiness. Awkward in hands and } \\
\text { legs, errors speaking, writing, put words on bad forms. All symptoms, especially mental, } \\
\text { worst after sleep or aggravation wakes him up from sleep. }\end{array}$ \\
\hline
\end{tabular}




\begin{tabular}{|c|c|}
\hline $\begin{array}{l}\text { Lycopodium } \\
\text { clavatum }\end{array}$ & $\begin{array}{l}\text { Lack of confidence, social timidity, runs away from difficulties. Hypersensitive, does mot } \\
\text { bear jokes, is always right. Stubborn, resentful, always defensive, dictatorial, desire of } \\
\text { domination, selfish and provocative, but coward with interested servility. Pleasant with } \\
\text { stronger or superiors, hard with weaker and inferior. Impressionable and scared by } \\
\text { authority or reprehension. Intolerant to contradiction, disobedient, opposition, jealous, } \\
\text { violent rage with aggressiveness; beats, preferentially, on the weaker. Violent rage, } \\
\text { speaks swearwords, threats, defiance, provokes. Proud, presumptuous, affected and looks } \\
\text { down on others. Weak children and too tired, lack of physical energy. Nervous tics, } \\
\text { enuresis, encopresis. Gastrointestinal symptoms, desire for sweets, chilly. Difficulty in } \\
\text { learning, errors reading and writing, unfit for mathematics. Fear of darkness, noises, } \\
\text { ghosts or imaginary things. Theme of the father in his role of educator; difficulties in } \\
\text { relationship with his father (Masi Elizalde). Precocious children, with appearance of } \\
\text { older, early white hair. }\end{array}$ \\
\hline $\begin{array}{l}\text { Mercurius } \\
\text { solubilis }\end{array}$ & $\begin{array}{l}\text { Problem in adaptation to the world: is resentful and socially marginalized, transgressor, } \\
\text { disrespectful, spites on the face of people, considers everybody as enemy. Restlessness, } \\
\text { agitation, awkward movements, quick speech, slow answers. Intolerance to contradiction, } \\
\text { angry attacks with violent impulses; destructive and aggressive against himself and } \\
\text { others, liable to become suicidal or homicidal, with desire to kill with knife - can stab } \\
\text { anyone, even his loved relatives. Remorse and anxiety of conscience by his acts - feels } \\
\text { guilty and is suicidal. Needs to dominate others; propensity to gambling, drugs and } \\
\text { alcohol. Liar, false, aggressive, selfish, accentuated destructiveness (auto, hetero and eco). } \\
\text { Wants to modify or destroy nature to rebuild it better- Theme of Perfection. Creativity: } \\
\text { always inventing something better (Masi Elizalde). It is one of the remedies characterized } \\
\text { by anxiety and haste, but his haste is unproductive, unlike Tarentula or Nux vomica that } \\
\text { present an efficient and productive activity. ADD with hyperactivity and impulsiveness, } \\
\text { aggressiveness, difficulty of understanding, memory and language - stammering. } \\
\text { Hypersexual excitability, with eroticism, lasciviousness, sexual crimes, rape. Constant } \\
\text { desire for change, journeys and to escape. Nervous affections after suppression of } \\
\text { secretions. Tendency to inflammation and ulceration in skin and mucous membranes. }\end{array}$ \\
\hline $\begin{array}{l}\text { Nitric } \\
\text { acidum }\end{array}$ & $\begin{array}{l}\text { Excessive physical irritability, impetuous, irritability by noises, irritable, out of himself by } \\
\text { nothing, with shivering. Is never cheerful or satisfied, runs away from contact, aversion to } \\
\text { be consoled, indifferent to the others, hard, without piety. Opposition and does not } \\
\text { hesitates to injure and to be rough. Irritated, stubborn, hateful and vindictive; with } \\
\text { inveterate habits, mischievous, impassioned with apologies. Theme of justice and mercy } \\
\text { - does not forgive and becomes inexorable no matter what the others say (Masi Elizalde). } \\
\text { Auto social marginalization that leads him to be corrupt, disorderly, and delinquent } \\
\text { conduct. Conduct disorder and oppositional defiant. Enuresis and bites the nails. } \\
\text { Tendency to warts. }\end{array}$ \\
\hline $\begin{array}{l}\text { Nux } \\
\text { vomica }\end{array}$ & $\begin{array}{l}\text { Physical, emotional and moral hypersensitiveness, also to noises, light, contradiction, pain } \\
\text { (faints from pain) - from any stimulus, with predominance of aggressiveness, not } \\
\text { measuring the consequences, unruly impulse. Irritable, jealous children, defiant, prudent } \\
\text { and zealous, inclined to be quarrelsome, vindictive, mischievous, cruel, nervous and } \\
\text { melancholic. They desire tranquility and loneliness, do not bear to be interrupted; do not } \\
\text { tolerate to be looked at. Worries with family, although may be rough. Impatient, } \\
\text { impetuous, insignificant disorders are unbearable; desire to kill himself with a knife. Any } \\
\text { innocent word offends him, hypersensitive to hurt self esteem (injured honor) - out of } \\
\text { himself, redness and shivering; impressionable by all external stimuli; sympathetic. } \\
\text { Theme of justice, of right and wrong (Masi Elizalde). ADD with hyperactivity and } \\
\text { impulsiveness. Disorder of language, stammering. Grabs people, wants to feel secure. }\end{array}$ \\
\hline
\end{tabular}




\begin{tabular}{|c|c|}
\hline Opium & $\begin{array}{l}\text { Mental excitability and agitation, exaltation of imagination and memory. Neurosensorial } \\
\text { hyper-excitability, startles by slight stimulus. In another phase can be numbed and } \\
\text { unfeeling. State of anesthesia or emotional and intellectual excitement, with joy, boldness } \\
\text { and sensation of invincibility. Aggravates from fear or fright that paralyzes him. The } \\
\text { emotional shock can trigger any illness or reaction. Aggressiveness, crises of violent rage; } \\
\text { liar (does not know what is true), jealous, malicious, cruel, wild, bites, says swearwords, } \\
\text { runs away, does not observe moral rules, aggravates from touch. Attacks of excessive } \\
\text { laughter, hysterical reactions, and sensation of having strong force, boldness, nocturnal } \\
\text { hyper-ideation. Indicated in autism and delirium. Enuresis, encopresis, convulsions after } \\
\text { fright or rage. }\end{array}$ \\
\hline Phosphorus & $\begin{array}{l}\text { Hypersensitive and vulnerable, with anxiety and fear of everything around him. Creative, } \\
\text { gifted, leader, affectionate. In the aggressive phase is agitated, violent, and disobedient, } \\
\text { does not accept orders, feels above the others. Proud, arrogant, liable to wild, violent rage; } \\
\text { strikes, spites, bites and says swearwords. Audacious, impetuous, impertinent, insolent, } \\
\text { quarrelsome. Impressionable, attracted to clairvoyance, intuitive, and artistic things. } \\
\text { Desire physical touch that ameliorates (magnetism), want to be hold to feel secure. Sexual } \\
\text { excitement and frequent masturbation. Disobedient, ADD with hyperactivity and } \\
\text { impulsiveness. Theme of light, clarity, fraternity. }\end{array}$ \\
\hline $\begin{array}{l}\text { Plumbum } \\
\text { metallicum }\end{array}$ & $\begin{array}{l}\text { Reading and speech disorders (loquacity / stammering), absentminded and restless } \\
\text { children, haste in acts and in answers, quarrelsome, irritable, attacks himself (liable to } \\
\text { stab himself) and others, lascivious, insolent and disrespectful, disobedient, surpasses his } \\
\text { limits, tendency to oppose and do prohibited things; excited by risky activities and } \\
\text { scandals, perverse and unfaithful. As compulsive gamblers may put at risk belongings } \\
\text { necessary to existence. Encopresis and nervous tics; depressive, progressively more } \\
\text { apathetic, paranoid (fear to be murdered). Problem with prohibition; does not accept } \\
\text { established limits by the law, does not accept prohibitions (Masi Elizalde). Can be } \\
\text { stuporous, slow in expression with depressed cerebral functions, with loss of memory, } \\
\text { especially for words. Patients become weak and emaciated quickly and excessively when } \\
\text { sick. States of sclerosis, neurological disorders, paralysis and atrophies (important action } \\
\text { on spinal marrow), and tendency to present intestinal problems. }\end{array}$ \\
\hline Staphisagria & $\begin{array}{l}\text { Hypersensitive to the slightest mental or moral impressions; to slightest action or innocent } \\
\text { word offends him, hypersensitive to the injured self esteem (injured honor), feels he is } \\
\text { unjustly treated and that does not deserve what they do to him. Great indignation } \\
\text { regarding things done by other persons or even by himself; afflicts himself with the } \\
\text { consequences. Disorders by repressed rage, with silent sorrow, or explosive rage throwing } \\
\text { objects. Ill-humored children that cry asking things and when receive them, sassy, move } \\
\text { or throw away. Sensitive to injustices suffered or imaginary, aggravates by reproach. If } \\
\text { insulted, is too dignified as to fight, controls rage and arrives at home ill, trembling and } \\
\text { exhausted; fear of losing self-control. Self-aggressive tendencies and to masturbation. } \\
\text { Theme of dignity and sexuality (Masi Elizalde). ADD with impulsiveness and } \\
\text { perturbations of conduct. Hypersensitive to injury. }\end{array}$ \\
\hline
\end{tabular}




\begin{tabular}{|c|c|}
\hline Stramonium & $\begin{array}{l}\text { Violent physical and intellectual agitation, intense loquacity. Irrational conduct, exalted } \\
\text { imagination, indifferent to pain. Sardonic, incoherent and incessant laughter, prayer, } \\
\text { pleads for, begs, squeaks, beats, bites, tears, scratches; does not bear staying alone, worse } \\
\text { in the dark and by loneliness. Hallucinations that frighten the patient and tries to escape; } \\
\text { imagines all sorts of things. The pupils dilate when infant is reproached; reddish and hot } \\
\text { face, with cold hands and feet; circumscribed redness of the cheeks. Stammering. } \\
\text { Convulsions. He curses, tears the clothes, exhibits himself, desires sto be naked, } \\
\text { conversation with angels. Intolerance to contradiction, foolish or ridiculous gestures, spites } \\
\text { on the face of people, cries alternating with laughter, dizzy laughter and foolish behavior. } \\
\text { Hallucinations with mice, snakes and other animal, sees devils; confused fantasies, } \\
\text { nocturnal terror. Seizes people to feel secure. Attacks of rage and fury alternating with } \\
\text { depression and violent acts. Answers with irrelevances, difficulty of concentration. } \\
\text { Theme of trash (the plant grows in the trash). }\end{array}$ \\
\hline Sulphur & $\begin{array}{l}\text { Cheerful, jovial, funny, sociable, however impulsive, impatient and bungler. Tired, } \\
\text { especially at } 11 \text { o'clock, indolent, aversion to physical efforts. Mental abstraction, ADD } \\
\text { with impulsiveness and conduct disorder, tendency to carelessness. Agitated, with } \\
\text { difficulty of learning and disturbs of expressive language. Encopresis, enuresis, grinds the } \\
\text { teeth and bites his own nails. Theme of merit, judges-itself worthy of all honors and glory } \\
\text { or that deserves nothing (Masi Elizalde). Wants to be seized by people to feel secure. } \\
\text { Lively, imaginative spirit, impatience, however selfish and lazy. }\end{array}$ \\
\hline $\begin{array}{l}\text { Tarentula } \\
\text { hispanica }\end{array}$ & $\begin{array}{l}\text { Physical and mental anxiety too intense, with great anxiety. Highly nervous children, } \\
\text { constant motion of the legs, arms, with incapacity to do anything; contractions and starts } \\
\text { of muscles. Does nott get keep quiet in any position; needs to keep walking, although } \\
\text { symptoms get worse. Hyperesthesia: the slightest excitement irritates him and is followed } \\
\text { by sorrow. Hysteria, with extreme sexual excitement up to mania; tendency to } \\
\text { masturbation and fondles genitals. Sensitive to songs, dance, sings, excessive joy. Crises } \\
\text { of violent rage and does not want to be touched; pulls out clothes, exhibitionist and } \\
\text { lascivious. Aggressive, perverse, sly, destructive tendency. Disobedient, does not obey } \\
\text { rules. Aversion to colors red, green, yellow or black. Great hyperactivity, without ADD, } \\
\text { but with conduct disorder. Enuresis. }\end{array}$ \\
\hline $\begin{array}{l}\text { Veratrum } \\
\text { album }\end{array}$ & $\begin{array}{l}\text { Always wants to be the first. Proud, extravagant, megalomaniac, liar, cheats, despises } \\
\text { others, insolent. Wants to be loved and accepted, embraces everybody. Ambitious, } \\
\text { employs any means to obtain what he desires. Aggravates by the loss of objects. Theme of } \\
\text { protection, of a position in the society, a high place; wants to be next to the powerful to } \\
\text { get protection (Masi Elizalde). Tendency to slander, critic, rude and rough, beats the feet } \\
\text { and does not admit contradiction. Conduct disorder accentuated and oppositional defiant. } \\
\text { Enuresis, encopresis, grinds the teeth during sleep and bites nails. Nervous tics. }\end{array}$ \\
\hline $\begin{array}{l}\text { Zincum } \\
\text { metallicum }\end{array}$ & $\begin{array}{l}\text { Patients with weak memory, aversion to work and to speak, lethargy and melancholy, } \\
\text { aversion to answer, repeats questions, reflects long before answering. Apprehensive fear } \\
\text { by an imaginary crime. Restless sleep and wakes up frightened by nightmares. Patients } \\
\text { with nervous and cerebral exhaustion, with absence of vitality, anemia and debility. } \\
\text { Depressive states and irritation of nervous system, convulsions. Disorders by suppressed } \\
\text { eruptions or discharges. Incessant and violently restless in the lower limbs and feet, also } \\
\text { during sleep. General anxiety, with automatic motions of mouth, arms and hands (tics and } \\
\text { straight). Lasciviousness, with masturbation and great sexual excitement, fondles } \\
\text { genitals. Makes mistakes in written and spoken expression, distracted and awkward, } \\
\text { imprudent and hasty, quarrelsome, malicious. Encopresis and enuresis. Problem with } \\
\text { force and power, lack of strength to arrive to his goal and remains in the middle of the } \\
\text { way; problems in the knees, which are the center of power (Masi Elizalde). }\end{array}$ \\
\hline
\end{tabular}

